\title{
Médiévales
}

Langues, Textes, Histoire

50 | printemps 2006

Sociétés nordiques en politique ( $\mathrm{XII}{ }^{\mathrm{e}}-\mathrm{XV}^{\mathrm{e}}$ siècles)

\section{La Saga de Thórdur kakali : une œuvre de propagande?}

Is the Thórdur kakali Saga a propaganda work?

\section{Einar Már Jónsson}

\section{(2) OpenEdition}

1 Journals

\section{Édition électronique}

URL : https://journals.openedition.org/medievales/1339

DOI : 10.4000/medievales.1339

ISSN : 1777-5892

Éditeur

Presses universitaires de Vincennes

\section{Édition imprimée}

Date de publication : 1 juin 2006

Pagination : 47-57

ISBN : 2-84292-186-0

ISSN : 0751-2708

\section{Référence électronique}

Einar Már Jónsson, «La Saga de Thórdur kakali : une œuvre de propagande? », Médiévales [En ligne], 50 I printemps 2006, mis en ligne le 15 septembre 2008, consulté le 24 avril 2022. URL : http:// journals.openedition.org/medievales/1339; DOI : https://doi.org/10.4000/medievales.1339

Ce document a été généré automatiquement le 24 avril 2022

Tous droits réservés 


\title{
La Saga de Thórdur kakali : une œuvre de propagande?
}

Is the Thórdur kakali Saga a propaganda work?

\author{
Einar Már Jónsson
}

1 Durant l'été 1250, un événement capital se passe en Islande. Thórdur kakali Sighvatsson, qui était revenu de Norvège en 1247 , avec l'appui du roi de Norvège, et avait vite obtenu le pouvoir sur l'ensemble de l'Islande, est rappelé par le roi et quitte le pays. Il ne devait jamais revenir en Islande, et ce fut en fait la fin de toute tentative sérieuse de la part de la famille des Sturlungar ${ }^{1}$ d'y obtenir la suprématie. Les années suivantes voient l'ascension progressive de son adversaire Gissur Thorvaldsson ${ }^{2}$ au milieu des troubles, et la mainmise du roi de Norvège, Hákon le Vieux, sur l'Islande.

2 S'il s'agit de toute évidence d'un tournant majeur dans l'histoire islandaise, la façon dont il est traité dans notre source principale sur les événements de cette période, la Sturlunga saga, paraît assez étonnante ${ }^{3}$. Le départ de Thórdur n'est ni raconté pour luimême ni mis en valeur en tant que fait marquant dans aucune des deux versions de la saga. Tel qu'il figure dans les éditions établies d'après la version du manuscrit Króksfjardarbók (version I) ${ }^{4}$, le texte porte sur les événements de l'été 1249 , la prédominance de Thórdur à l'althing (parlement), la lettre du roi Hákon qui le convoque en Norvège, et son conflit avec l'évêque Heinrekur ${ }^{5}$, qui s'est rendu auprès du roi en portant des accusations contre Thórdur. Pour finir, la saga précise qu'en Norvège peu de gens prennent alors la défense du chef islandais. À cet instant du récit, on s'attendrait à ce que soit raconté l'événement principal de l'année suivante, le départ de Thórdur ; à l'inverse, la saga revient en arrière et entreprend de relater une dispute entre chefs dans l'Islande du sud-est. Ce conflit, d'une importance purement locale, avait commencé au début des années 1240, et il fut soumis à l'althing de 1250, où Thórdur était toujours le maître du jeu. Il y eut un jugement, qui au fond ne réglait rien. Selon la saga, Thórdur rentre ensuite chez lui après le parlement, le même été il part pour la Norvège, et les gens estiment qu'il a mal réglé cette affaire (chap. 215 [365]). Puis, la saga continue à raconter les suites de cette affaire locale qui se poursuit jusqu'en 1252. En somme, le départ de Thórdur en 1250 apparaît comme un détail 
secondaire dans «la saga des gens de Svínafell », comme on appelle cette partie de la Sturlunga saga, où son action, dans ce cas précis, est d'ailleurs mal jugée.

Une lacune étrange

3 Cette façon de traiter l'événement est pourtant en opposition criante avec son importance réelle. Pour essayer de le comprendre, il faut regarder de plus près la nature de notre source, la Sturlunga saga, et plus précisément la partie de cette œuvre portant sur Thórdur kakali. Comme on le sait, la Sturlunga saga est une vaste compilation effectuée au début du XIve siècle par un compilateur qui avait devant lui de nombreuses sagas sur les événements du siècle précédent et qui les a copiées en les juxtaposant et en les combinant de diverses façons. La "colonne vertébrale ", si l'on peut dire, de cette compilation est constituée par la Sturlu saga, la saga de Sturla de Hvammur, l'ancêtre des Sturlungar, et l'Íslendinga saga, la Saga des Islandais, de Sturla Thórdarson ${ }^{6}$; mais à ces deux textes le compilateur en a ajouté plusieurs autres, en essayant de respecter autant que possible l'ordre chronologique des événements. Le compilateur explique sa méthode et indique quelques-unes de ses sources dans une courte note (parfois appelée " préface ") qu'il insère à la fin de Sturlu saga ${ }^{7}$. En dehors de la Saga des Islandais, aucun des textes cités ne va plus loin que 1215 environ, après cette date le compilateur donne l'impression de suivre exclusivement cette dernière.

Il a cependant été démontré depuis longtemps ${ }^{8}$ qu'après le chapitre 163 [308] de la Saga des Islandais, le compilateur met momentanément de côté l'œuvre de Sturla Thórdarson et suit une autre source pour les événements survenus de 1242 à 1249, qui occupent les chapitres 164 à 211 [309 à 357]. Ce changement n'est pas signalé, mais la différence entre les deux sources est manifeste. Si Sturla Thórdarson ne cherche aucunement à se mettre personnellement en valeur dans sa Saga des Islandais, et s'efforce d'écrire une histoire globale d'Islande pendant la période qui l'occupe, on constate qu'il est par la force des choses le mieux informé sur les événements - et ils étaient nombreux auxquels il avait assisté lui-même, et que par conséquent la saga a tendance à le suivre et à refléter son regard. À partir du chapitre 164 [309] donc, la perspective change. La saga est entièrement centrée autour de Thórdur kakali et de son action, tout est raconté de son point de vue, et si cela peut se justifier par le fait qu'il était mêlé à la plupart des événements de cette époque, on a remarqué que la saga passe sous silence des faits importants mais qui ne concernent pas son histoire ${ }^{9}$. Si Sturla Thórdarson joue toujours un rôle de premier plan, il devient maintenant un personnage comme les autres, la saga n'est pas racontée de son point de vue ; mais plutôt de celui des fils d'un certain Dufgus, partisans de Thórdur kakali, notamment Svarthöfdi Dufgusson, qui est le plus important informateur des chapitres 164 à 211, s'il n'en est pas lui-même l'auteur. Sont mentionnés en effet des épisodes que Svarthöfdi était le seul à pouvoir raconter (ou à avoir eu l'idée de raconter) ${ }^{10}$. Après le chapitre 211, la perspective change de nouveau, lorsque la saga se tourne vers le conflit dans le sud-est, et il est évident que le compilateur change encore de source, toujours sans le signaler.

5 Pour cette raison, les spécialistes ont pris l'habitude d'appeler les chapitres 164 à 211 la Thórdar saga kakala, la "Saga de Thórdur kakali », un titre qui semble s'imposer même s'il n'est pas donné par les sources médiévales, et on s'est demandé quelle était sa forme originelle et comment le compilateur l'avait éventuellement coupée ou abrégée pour la faire entrer dans sa compilation. Dans la Sturlunga saga, on trouve plusieurs sagas consacrées à un individu de premier plan; il y a notamment Sturlu saga, Gudmundar saga dýra et Hrafns saga Sveinbjarnarsonar. Chacune de ces sagas commence 
par une présentation de la généalogie du héros et raconte ensuite sa vie du début jusqu'à sa mort. Dans la compilation, on peut constater cette manière de procéder dans les deux premières sagas, que le compilateur a utilisées intégralement. En ce qui concerne la Hrafns saga, elle commence lorsque Hrafn revient d'un voyage en Norvège où il avait accompagné l'évêque Gudmundur, mais cette saga est conservée ailleurs dans sa version originale, et on peut donc constater qu'elle racontait la vie de son héros depuis le début, elle aussi, et que le compilateur en a supprimé plus de la moitié. On a donc souvent pensé que le cas de la Thórdar saga kakala devait être analogue : dans sa version intégrale elle aurait raconté la vie de Thórdur depuis sa naissance jusqu'à sa mort en Norvège en 1256, et le compilateur aurait donc coupé tout le début de la saga, ainsi que le récit des années 1250 à 1256. D'autres spécialistes, en revanche, estiment qu'en réalité la Thórdar saga commençait seulement lorsque Thórdur revint de Norvège pour la première fois en 1242, ce qui est raconté dans le chapitre 164. Selon Björn M. Olsen, la Thórdar saga aurait ainsi été écrite comme une continuation de la Saga des Islandais, qui se serait arrêtée en $1242^{11}$. Mais il semble maintenant établi que celle-ci continuait au moins jusqu'en 1255, sinon plus loin encore, et que la partie de la Sturlunga saga qui traite des années postérieures à 1252 en provient. Pétur Sigurdsson a donc pensé que l'auteur de la Thórdar saga a pris la plume car il estimait que le récit de Sturla Thórdarson pour les années 1242 à 1250 (qui n'a pas été conservé, s'il a jamais existé) n'accordait pas assez d'importance à son héros. Peut-être la saga couvrait-elle seulement ces années-là, les années où Thórdur kakali joua effectivement un rôle de premier plan dans l'histoire islandaise ${ }^{12}$.

6 Ces théories ont toutes une chose en commun: quelle que soit l'opinion que les spécialistes puissent avoir sur la longueur et l'étendue originelles de la Thórdar saga, celle-ci devait forcément raconter en détail le départ de Thórdur en 1250. C'est un épisode capital si la saga raconte toute la vie de Thórdur, car c'est un tournant définitif dans sa carrière, et c'est aussi un épisode capital si la saga ne raconte que les agissements de Thórdur entre 1242 et 1250, car il en marque la fin. Dans les deux cas, il est incompréhensible que le compilateur ait supprimé le passage sur le départ de Thórdur, d'autant plus qu'il cherche toujours à utiliser pour sa compilation le passage le plus détaillé et le plus précis de ses sources. Pour certains commentateurs de la Sturlunga saga, son compilateur aurait quitté la Thórdar saga « trop tôt ${ }^{13}$ » - c'est-à-dire avant le départ de Thórdur -, pour pouvoir raconter les événements de chaque année, éloignés les uns des autres et indépendants, dans un ordre chronologique. Ainsi, il aurait eu l'intention de parler du départ de Thórdur à sa juste place dans la chronologie, au milieu du récit sur le conflit dans le sud-est. Mais le compilateur, qui se rend bien compte des difficultés rencontrées quand on veut rassembler et harmoniser les récits séparés d'événements contemporains sans rapport entre eux ${ }^{14}, n^{1}$ hésite pas à faire de petites entorses à la chronologie. Et s'il a eu l'intention de raconter le départ de Thórdur à sa place exacte, pourquoi ne l'a-t-il pas fait? Cette lacune dans l'histoire de Thórdur est à tel point criante que l'auteur de la version du manuscrit Reykjafjardarbók (la version II de la Sturlunga saga) l'a bien sentie et qu'il a inséré, en plein milieu du récit du conflit dans le sud-est, un chapitre relatant de façon succincte le départ de Thórdur et les arrangements qu'il avait pris auparavant (chapitre $213{ }^{15}$ ). Mais ce chapitre ne figure pas dans la version I, il ne se trouvait pas dans le prototype des deux versions et Pétur Sigurdsson a démontré qu'il ne peut en aucun cas provenir de la Thórdar saga. Il a probablement été écrit au XIV siècle par l'auteur de la version II qui a puisé dans diverses sources, dont certaines peuvent être identifiées ${ }^{16}$. Par rapport 
aux théories habituelles sur la version primitive de la Thórdar saga et sur la façon du compilateur de traiter ses sources, l'énigme reste donc entière.

Quel était le dessein de l'auteur de la Saga de Thórdur?

7 Je vais ici tenter de proposer une explication qui me paraît avoir une certaine importance pour l'idée qu'on peut se faire de la véritable nature de la Thórdar saga : si le compilateur n'a emprunté à cette saga aucun passage sur le départ de Thórdur en 1250, c'est qu'un tel passage n'existait pas. La saga ne racontait pas le départ de Thórdur, elle s'arrêtait avant.

Pour éclaircir cette hypothèse, il faut revenir au début des chapitres sur Thórdur dans la Sturlunga saga et leur manière de présenter les faits. Comme certains spécialistes l'ont souligné ${ }^{17}$, le chapitre 164 [309] représente un nouveau départ, il reprend les mêmes événements que les chapitres précédents, notamment le chapitre 163 [308], mais au lieu de les raconter d'une façon chronologique comme les chapitres précédents l'avaient fait, il en brosse un tableau synchronique. Si le chapitre 164 avait été situé, dans l'éventuelle version primitive de la Thórdar saga, en plein milieu d'un exposé historique, cet exposé aurait évidemment dû raconter les faits dans leur véritable ordre chronologique. En revanche, tel qu'il est, ce tableau se présente, non pas comme une partie d'un récit historique, mais comme une introduction à un récit. Cet objectif ressort également des premières phrases du chapitre 164 : «Un hiver après la mort de Snorri Sturluson commencèrent les événements qui ont entraîné maintes conséquences par la suite ».

9 En fait, ce tableau résume la situation en Islande exactement comme elle pouvait apparaître aux yeux de Thórdur, lorsqu'il débarqua de son bateau à l'automne 1242 après quelques années d'absence. Le chapitre suivant (165 [310]) le précise encore : il raconte les rapports de Thórdur avec ses proches et son état psychologique dans la situation désespérée où il se trouvait. La première phrase est donc un véritable incipit, et si les deux chapitres 164-165 sont le début d'un récit, on ne peut guère les qualifier de début de saga au sens classique du terme ${ }^{18}$.

La suite raconte les combats difficiles de Thórdur. Il était en fait un des derniers survivants d'une cause qui paraissait définitivement perdue. La famille des Sturlungar, à laquelle il appartenait, avait subi deux graves défaites, d'abord dans la bataille d'Örlygsstadir, où le père et quatre des frères de Thórdur avaient trouvé la mort, ensuite avec l'assassinat de Snorri Sturluson. En 1242, l'Islande était en fait divisée entre ses ennemis, Kolbeinn le Jeune et Gissur Thorvaldsson, qui était en Norvège mais qui avait laissé un représentant en Islande. Thórdur est obligé de les affronter, mais dans une situation de grande faiblesse. Dans le récit que font les chapitres 164 à 211 des événements ultérieurs, deux choses doivent être mises en évidence.

11 Tout d'abord la manière dont la saga présente les ennemis de Thórdur. Elle prend soin de rendre justice à leurs qualités et à leur vaillance, et fait même des digressions pour les souligner. Kolbeinn le Jeune ne participe guère à la bataille navale de Húnaflói, mais c'est à cause de sa maladie, précise la saga, qui ajoute: "Mais tous les gens savaient qu'il était l'homme le plus valeureux » (ch. 193 [339]). On a aussi observé que le dernier grand adversaire de Thórdur kakali, Brandur Kolbeinsson, vaincu dans la bataille de Haugsnes et exécuté sur l'ordre de Thórdur, est toujours présenté sous une lumière favorable. Cependant, on remarque qu'en général la saga a aussi tendance à trouver des excuses aux actes de violence commis par Thórdur et par ses hommes, en attribuant par exemple aux victimes divers méfaits envers la famille de Sturlungar - parfois des 
méfaits qu'ignore la « Saga des Islandais » ou qui sont même en contradiction flagrante avec cette dernière saga - et en présentant ainsi les actes de Thórdur comme une vengeance légitime. Ainsi, lorsque Thórdur s'installe à deux reprises dans la ferme d'un homme riche et puissant, Ormur Bjarnason, en puisant à coup sûr largement dans ses biens, la saga précise que ce dernier avait «pris une part importante au meurtre de Snorri Sturluson» (ch. 171 [315]). Mais, selon la Saga des Islandais, ce même Ormur Bjarnason n'avait justement pas voulu participer à l'attaque contre Snorri et avait quitté le groupe des assaillants pour rentrer chez lui, dès qu'il eut compris leurs intentions (Saga des Islandais, ch. 151 [301]) ${ }^{19}$. En ce qui concerne certains méfaits commis par les hommes de Thórdur, la saga dit que tout le monde sentait que Thórdur lui-même en était mécontent (chap. 177 [321]), et il finit par chasser un dénommé Ásbjörn Gudmundarson, celui de ses «lieutenants » qui était responsable des pires méfaits. La saga souligne à deux reprises que Thórdur ordonna à ses hommes d'épargner les femmes et de respecter toujours le droit d'asile dans les églises - il fit même le serment solennel de le faire (chap. 181 [326] et 182 [327]. Mais les homicides, somme toute analogues, perpétrés par ses adversaires sont souvent décrits comme des crimes gratuits (voir les chapitres 186-188 [331-334, les deux versions sont assez divergentes]). La saga souligne également que Kolbeinn le Jeune commit une action sans précédent en Islande : il fit brûler des baleines échouées sur la côte afin qu'elles ne servent pas de nourriture à Thórdur et à ses hommes (ch. 197 [342]). Et à plusieurs reprises on attribue à Kolbeinn le Jeune l'idée inouïe de dévaster de fond en comble les fjords de l'ouest, où Thórdur avait sa base, pour l'empêcher définitivement d'y trouver asile (ch. 175 [319], 176 [320], 195 [341], 197 [343]).

D'autre part, on trouve parfois dans la saga des jugements, des remarques prémonitoires ou des prophéties, présentés comme l'expression de l'opinion publique, de telle sorte qu'on ne les remarque guère. Quand Thórdur entreprend de rallier les bœendr ${ }^{20}$ à sa cause, il y a d'abord ce jugement ambigu : "Lorsque Thórdur eut fini son discours, les gens avaient l'impression qu'il deviendrait un homme sensé, lorsqu'il arriverait à freiner sa violence. On trouvait qu'il parlait avec une certaine raideur au début, mais au fur et à mesure qu'il continuait son discours et que les auditeurs devenaient plus nombreux, ses propos se faisaient plus hardis et plus éloquents » (ch. 167 [312]). Après sa fuite échevelée à travers les Mýrar en plein hiver, où il échappe de justesse à ses ennemis, l'auteur de la saga remarque (ch. 173 [317]) : «Tout le monde avait l'impression que si Thórdur s'était échappé, c'était qu'il était destiné à réaliser quelque chose de grand ». Un peu plus tard, dans des circonstances qui ne semblent pas justifier une telle remarque, la saga souligne (ch. 182 [327]) : « Tous ceux qui avaient participé à cette expédition avec Thórdur pensèrent qu'il deviendrait un très grand chef, s'il se maintenait sain et sauf ». Après la bataille navale dans le Húnaflói, l'auteur de la saga attribue à Kolbeinn le Jeune des paroles prémonitoires dont il est absurde d'imaginer qu'il les ait prononcées dans les circonstances telles qu'elles sont décrites. Il dit en effet: "J'ai l'impression que dans cette bataille il y a eu un renversement de fortune (hamingjuskipti) entre Thórdur et moi » (ch. 195 [341]). En réalité, la bataille navale était une défaite de Thórdur. Le "renversement de fortune » n'est intervenu que plus tard, et il était dû à la mort prématurée de Kolbeinn le Jeune (de maladie) et au fait qu'après la mort de celui-ci Thórdur n'avait plus d'adversaire à sa hauteur présent dans le pays.

Dans ces passages, on a l'impression que l'auteur de la saga est en train de raconter les débuts d'une personne promise à grande destinée - une destinée qui correspond à de 
telles prémonitions - et qui marche inexorablement vers la victoire définitive. Il est tentant de rapprocher ces remarques avec des passages de la première partie de la Saga de Sverrir, qui provient de Grýla, une œuvre de propagande justement, écrite du vivant de Sverrir et destinée à justifier ses actions ${ }^{21}$. Dans cette œuvre, il ne s'agit pas de prophéties mais d'interprétations de "signes " post eventum. À propos des agissements de Sverrir dans son enfance, la saga dit ceci (ch. 1): «En sachant par la suite quel homme il est devenu, les gens estimaient qu'il y avait eu beaucoup de signes indiquant qu'il avait une grande nature ${ }^{22}$." Et à propos d'un événement passablement miraculeux quelques années plus tard, la saga donne cette interprétation (ch. 13) : « Il est maintenant évident pour tous, que ce radeau portait l'homme destiné à réaliser des hauts faits qui ne s'étaient pas encore produits et à occuper une position plus élevée qu'il n'avait alors, puisque le radeau flottait avec les hommes mais sombra dès qu'ils eurent débarqué ${ }^{23}$ ". évident que la suite des événements n'a pas justifié les anticipations de sa saga. Thórdur n'a établi sa domination en Islande que pendant une courte période, ensuite sa carrière a tourné court, et s'il a donné de grandes promesses, il ne les a jamais réalisées. Il y a cependant eu un court moment historique où on pouvait penser que son heure était réellement venue, lorsque, ayant vaincu tous ses adversaires, il semblait désormais installé comme le maître absolu de l'Islande pour l'avenir, malgré une résistance dans le sud de l'île. C'était pendant les années 1248-1249.

Mon hypothèse est donc la suivante: ce qu'on appelle traditionnellement la Thórdar saga n'est pas la «Saga de Thórdur » au sens habituel de "saga ». Il s'agit plutôt d'une œuvre de propagande destinée à justifier l'action de Thórdur et à le présenter sous un jour favorable. Écrite lorsque Thórdur était au sommet de sa puissance, elle s'arrêtait à ce moment-là ${ }^{24}$. On peut alors se demander pourquoi ce texte donne parfois une image favorable de certains des adversaires de Thórdur et pourquoi il ne cache pas des côtés moins positifs de Thórdur lui-même. En fait cette œuvre doit être replacée dans un contexte particulier qui jette une vive lumière sur elle. Lorsque Thórdur et Gissur se trouvèrent en Norvège en 1246 et présentèrent le différend qui les opposait devant le roi, Thórdur fit lire un long texte, appelé rolla, c'est-à-dire " rôle » ou « rouleau », qu'il avait fait écrire au sujet des démêlés des Sturlungar avec les Haukdælir, la famille dont Gissur était le chef, et qui précisait entre autres « les nombreuses pertes humaines que Thórdur avait subies ». Gissur dit qu'il avait des réponses à tout cela même s'il n'avait pas mis ses propres récits par écrit, mais il ajouta, de manière particulièrement significative, qu'il estimait que Thórdur avait honnêtement exposé les relations entre les deux familles (ch. 208 [354]).

Le texte qu'on appelle traditionnellement Thórdar saga n'est certainement pas celui que Thórdur kakali fit lire devant le roi Hákon le Vieux, il est beaucoup plus long et destiné à un autre public. Mais la facture de ce "rôle", que la remarque de Gissur reflète indirectement, montre que les "propagandistes » de Thórdur, si l'on peut les appeler ainsi, estimaient que pour être efficace une œuvre de propagande devait respecter les faits et avoir au moins l'aspect de la neutralité. C'est dans cet esprit que la Thórdar saga a été écrite : on pourrait donc la considérer comme une œuvre de propagande sous la forme d'une saga. L'auteur a si bien réussi que, grâce à cette saga, les Islandais ont toujours gardé une image très positive de Thórdur kakali. 
Mais, pour les historiens d'aujourd'hui ce texte peut indiquer un usage méconnu de l'écriture des sagas au Moyen Âge, car le cas de la Thórdar saga n'est pas unique. On a en effet remarqué que la première saga de la Sturlunga saga, celle de Thorgils et de Haflidi, avait un contenu moral et politique évident ${ }^{25}$. Si elle raconte l'histoire d'un grave conflit entre deux chefs importants dans les années 1117-1121, c'est pour insister sur la nécessité d'éviter les conflits et de maintenir la paix. Écrite peut-être vers 1237, elle s'adresse en somme aux chefs contemporains pour leur dire: "Arrêtez de vous battre, avant que la situation ne s'aggrave encore, et essayez plutôt de résoudre pacifiquement vos querelles comme Thorgils et Haflidi ont su le faire ». La recherche de la paix dans la société islandaise dans la première moitié $\mathrm{du}$ XIII ${ }^{\mathrm{e}}$ siècle s'insère donc, finalement, au cœur du discours politique véhiculé par la Sturlunga saga, qui pourtant résonne de furieuses et sanglantes batailles. En ce qui concerne une autre branche de sagas, celles dites « des Islandais », il a été suggéré également que la Saga de Thórir aux poules avait été écrite en relation avec un débat au parlement islandais de 1281 à propos d'une nouvelle loi permettant des réquisitions de foin en période de graves intempéries, afin d'aider les paysans qui en manquaient à nourrir leur bétail ${ }^{26}$. Cette saga semble en effet avoir été composée dans le but de justifier la nécessité d'une telle loi. Ainsi une étude plus systématique des rapports éventuels entre les sagas et les vicissitudes politiques qui entouraient l'époque de leur rédaction pourrait se révéler prometteuse.

Généalogie simplifiée des Sturlungar

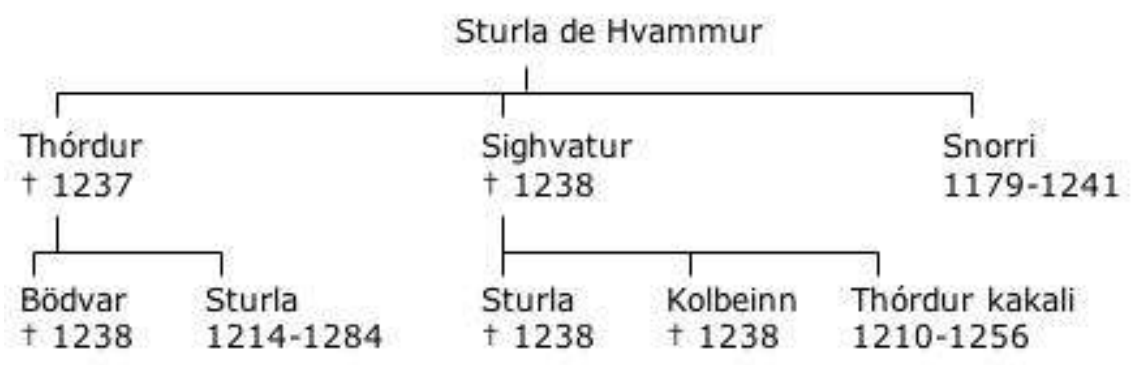

\section{NOTES}

1. Une des puissantes familles islandaises qui revendiquaient le pouvoir à la fin $d u X I I^{e}$ siècle et dans la première moitié du xiII ${ }^{\mathrm{e}}$ siècle ; elles entretinrent entre elles d'âpres rivalités, dont le récit constitue la matière principale de la Sturlunga saga. Voir en fin d'article une généalogie simplifiée des Sturlungar.

2. Gissur Thorvaldsson (1209-1268) appartenait à la famille des Haukdœlir.

3. Pour la Sturlunga saga, nous avons utilisé l'édition de J. JóHANNESSON, M. FinNBOGASON et K. ELDJÁRn, Reykjavík, 1946 (dite « l'édition de Reykjavík ») et celle d'Ö. THORSSON et al., Reykjavík, 1988 (dite « l'édition de Svart á hvítu»). En accord avec la tradition savante nous employons la numérotation des chapitres de la « Saga de Thórdur » qui 
remonte à Gudbrandur Vigfússon au XIX ${ }^{e}$ siècle et qui est donnée entre parenthèses dans « l'édition de Reykjavík », mais nous indiquons aussi, entre crochets, les numéros des chapitres dans « l'édition de Svart á hvítu », utilisés dans la traduction de R. BOYER, La Saga des Sturlungar, Paris, 2005. Voir « Sturlunga saga », dans Medieval Scandinavia. An Encyclopedia, P. Pulsiano et K. Wolf éd., New York-Londres, 1993, p. 616-618.

4. Nous verrons plus tard les problèmes particuliers de la « version II » (manuscrit Reykjafjardarbók).

5. Évêque de Holar (1247-1260) ; il était d'origine norvégienne.

6. Sturla Thórdarson (1214-1284) était le cousin germain de Thórdur. Voir « Sturla Thórdarson », dans Medieval Scandinavia. An Encyclopedia, op. cit., p. 613-614.

7. La Saga des Sturlungar, op. cit., trad. R. BOYER, p. 97-98.

8. Voir par exemple B. M. ÓLSEN : «Um Sturlungu », Safn til sögu Íslands og íslernzkra bókmennta, III, 1902, p. 437-447.

9. Cf. P. SIGURDSSON, Um Íslendinga sögu Sturlu Thórdarsonar, Reykjavík, 1933-1935, p. 91.

10. B. M. ÓLSEN, loc. cit., p. 447-462.

11. B. M. ÓLSEN, loc. cit., p. 467.

12. P. SIGURDSSON, op. cit., p. 91.

13. L'expression est de P. SiguRDSSON, op. cit., p. 93-94, cf. aussi p. 97-98.

14. Voir la remarque dans la "préface » : « De nombreuses sagas ici présentes se passent simultanément, on ne peut toutefois pas les écrire en même temps » (La Saga des Sturlungar, op. cit., trad. R. BOYER, p. 98).

15. Publié en appendice dans l'édition de Reykjavík, II, 288-289, et également dans celle de « Svart á hvítu », II, 934-935.

16. P. SIGURDSSON, op. cit., p. 92-94.

17. Déjà B. M. ÓLSEN en 1902, loc. cit., p. 467.

18. Une saga commence presque toujours pas une généalogie et une présentation des personnages.

19. Voir, pour d'autres exemples, B. M. ÓLSEN, loc. cit., p. 444-445, et les chapitres 179 [324] et 180 [325] de la Thórdar saga.

20. Les bœendr (singulier bóndi) sont les paysans libres. Ils participent à l'althing (parlement).

21. Sverris saga etter Cod. AM 327, $4^{\circ}$, G. INDREB $\varnothing$ éd., Kristiana, 1921 (nouvelle édition en fac-similé, Oslo, 1981). Sverrir, ou Sverre Sigurdsson, roi de Norvège (1177-1202). Voir "Sverris saga », dans Medieval Scandinavia. An Encyclopedia, op. cit., p. 628-629; et aussi, S. BAGGE, « La Sverris saga, biographie d'un roi de Norvège », Proxima Thulé, t. 2, 1996, p. $113-128$.

22. Sverris saga, op. cit., p. 3.

23. Ibid., p. 13.

24. Ce n'est pas notre propos ici d'étudier le problème de la fin de la Saga de Thórdur. Le dernier chapitre commun aux deux versions de la Sturlunga saga est le chapitre 208 [354] qui raconte le séjour de Thórdur et de Gissur en Norvège en 1246-1247, avant le retour de Thórdur, mandaté par le roi. Il est suivi, dans la version I, par les chapitres 209-211, qui mêlent le récit du retour à des repères chronologiques et des résumés de la situation. Certains passages pourraient appartenir à la Thórdar saga, tandis que d'autres portent la trace du compilateur. La version II n'a rien qui corresponde à ces trois chapitres, en revanche elle contient trois chapitres différents, insérés dans la Saga des gens de Svínafell (dont le « chapitre 213 » déjà étudié) et qui ne peuvent pas provenir de 
la Thórdar saga (voir P. SIGURDSSON, op. cit., p. 94-96). On pourrait peut-être penser que la Thórdar saga se terminait par des passages que la suite des événements avait rendus plus ou moins obsolètes, et que les auteurs des deux versions ont essayé de raccommoder cette fin, chacun à sa façon. En ce qui concerne le récit de la mort de Thórdur (chap. 192 [477]), que certains éditeurs modernes attribuent à la Thórdar saga, rien n'autorise à le faire ; il ne se trouve que dans la version II et provient peut-être de la Saga des Islandais, comme le pensent les éditeurs de l'édition de Reykjavík, ou d'une autre source encore.

25. Voir " édition de Reykjavík », II, p. XXIV-XXV.

26. Hæensa Póris saga, dans Borgfirðinga sögur, S. NORDAL et G. JónSSON éd., Reykjavik, 1938 (Íslenzk Fornrit, 3), p. XXX-XXXI.

\section{RÉSUMÉS}

Il est couramment admis que parmi les sources de la Sturlunga saga il y avait une "Saga de Thórdur kakali », dont le compilateur aurait coupé le début et la fin pour ne garder que la partie concernant les années 1242-1250. L'article propose une autre hypothèse sur ce texte : il ne serait pas un fragment d'une saga au sens courant de ce mot, mais un écrit politique de circonstance destiné à expliquer l'action de Thórdur, membre de la puissante famille des Sturlungar et à justifier sa domination sur l'Islande. Composé lorsque Thórdur était au sommet de sa puissance, cet écrit se serait arrêté avant son départ pour la Norvège en 1250, qui sonna le glas de ses ambitions politiques.

Is the Thórdur kakali Saga a propaganda work? This paper proposes a theory about the «Saga de Thórdur kakali » which is a part of the huge compilation Sturlunga saga. According to the author, this text, which the compilator follows for the years 1242-1250, was not a saga telling the whole life of its hero untill his death in 1256, but a political pamphlet written to explain the action of Thórdur and to justify his domination over Iceland. It was written when he was at the height of his power and stopped before his departure from Iceland in 1250, which put an end to his ambition.

\section{INDEX}

Mots-clés : aristocratie islandaise, conflits de pouvoir, saga, propagande politique

Keywords : Icelandic aristocracy, power conflicts, political propaganda

\section{AUTEUR}

\section{EINAR MÁR JÓNSSON}

Université Paris IV Sorbonne, UFR d'Études germaniques, département d'études nordiques, Centre universitaire Malesherbes, 108, bd Malesherbes, 75017 Paris 